\title{
Tradução, equivalência semântica e adaptação cultural do Marijuana Expectancy Questionnaire (MEQ)
}

\author{
Rosemeri Siqueira Pedroso ${ }^{1}$ \\ Margareth da Silva Oliveira \\ Renata Brasil Aranjo \\ João Feliz Duarte Moraes
}

\begin{abstract}
Resumo
O objetivo desse estudo foi traduzir, adaptar culturalmente e verificar a equivalência semântica do Marijuana Expectancy Questionnaire (MEQ), o qual avalia as crenças em relação ao uso de maconha, podendo ser importante no tratamento e prognóstico dos dependentes químicos desta substância. O MEQ foi traduzido do inglês para o português, aplicado em 10 sujeitos e submetido ao brainstorming num grupo de 4 sujeitos para reprodução individual e verbal, item a item. Realizou-se o back-translation, uma versão para o idioma de origem, a partir da primeira tradução e do brainstorming. Logo após, traduziu-se novamente para o português. Todo o processo foi analisado por um comitê de juízes especialistas, os quais emitiram pareceres com as observações pertinentes. Realizou-se a análise descritiva interjuízes, verificando-se freqüências e porcentagens. Considerando-se os pareceres dos especialistas, construiu-se então a versão final do MEQ - adaptação brasileira.

Palavras-Chave: Equivalência semântica; Expectativas; Maconha; Questionário.
\end{abstract}

\section{Translation, semantic equivalence and cultural adaptation of Marijuana Expectancy Questionnaire (MEQ)}

\begin{abstract}
The objective of this study was to translate, to adapt culturally and verify the semantic equivalence of the Marijuana Expectancy Questionnaire (MEQ), which evaluates the beliefs to the marijuana's use, which may be important in the treatment and prognostic of the chemical dependents of this substance. The MEQ was firstly translated from English to Portuguese, administered to 10 persons, and then submitted to brainstorming in a group of 4 persons for individual and verbal reproduction, item by item. Back-translation was executed based on first translation and from brainstorming to the origin language. Soon after, it was translated again into Portuguese. All the process was analyzed by a committee of specialists, which emitted a decision and the pertinent comments. The descriptive judges' analysis was done verifying frequencies and percentages. Considering the decision of the specialists, the final version of the MEQ (Brazilian Adaptation) was constructed.

Keywords: Semantics equivalence; Expectations; Marijuana; Questionnaire.
\end{abstract}

As expectativas de resultados sobre os efeitos de determinada droga são crenças cognitivas, culturais e pessoais que podem levar o sujeito a engajar-se no comportamento aditivo. Há evidências de que os efeitos subjetivos e comportamentais de outras drogas, além do álcool, são influenciados pelas expectativas, daí a importância de se desenvolver e validar instrumentos que mensurem, especificamente, as expectativas de resultados relacionadas ao uso da maconha (Marlatt \& Gordon, 1993).

A maconha, como droga que altera o humor, destaca-se pelo seu freqüente uso entre os jovens. Laranjeira, Jungerman e Dunn (1998), referem que os efeitos prazerosos da maconha são: a sensação de relaxamento, os cinco sentidos mais aguçados, a diversão a partir de qualquer coisa, a euforia e o aumento do prazer sexual. Quanto aos efeitos adversos temos: a ansiedade, o pânico, a paranóia, a diminuição das habilidades mentais (especialmente, da atenção e memória), a diminuição da capacidade motora e o aumento do risco de ocorrerem sintomas psicóticos.

Brown (1993) revisou a construção e o papel das expectativas no desenvolvimento do abuso de substâncias. Considerou que as expectativas do efeito das drogas são fatores importantes na etiologia da adição, sendo que as expectativas para outras drogas que não o álcool deveriam ser estudadas e conhecidas. A autora avaliou o envolvimento de cada expectativa no processo de mudança do comportamento aditivo. Abordou ainda as expectativas de resultados a partir de três questionamentos: Qual a expectativa do efeito da droga? Como as expectativas se relacionam com o desenvolvimento do comportamento aditivo? Como

${ }^{1}$ Endereço para correspondência:

Av. João Pessoa, 211/1.102 - Bairro Farroupilhas - 90040-000 - Porto Alegre-RS

E-mail: rosemeripedroso@yahoo.com.br 
incorporar as expectativas na prevenção e intervenção em casos de adição, a partir de novos comportamentos?

As crenças têm sido estudadas como indicadoras dos sentimentos das pessoas diante de suas experiências e o quanto isso se liga aos efeitos. Torna-se necessário saber como as crenças são adquiridas e sustentadas e, assim, saber como podem ser modificadas (Eiser, 1997).

Oliveira, Szupzsynski, Camilo e Faggiani (2003), realizaram um estudo transversal, com 24 adolescentes usuários de drogas, com idades entre 13 e 18 anos (média de 16,6 anos), sendo 91,7\% ( $\mathrm{n}=22)$ do sexo masculino. Os instrumentos utilizados foram: uma entrevista estruturada, com dados sociodemográficos, e um questionário com 20 itens sobre crenças habituais a respeito do consumo de drogas (Beck, Wrigh, Newman \& Liese, 1993). Quanto às crenças cognitivas avaliadas, 91,7\% ( $\mathrm{n}=22)$ acreditavam que o seu abuso da substância incomodava as outras pessoas e $41,66 \%(n=10)$ diziam não conseguir relaxar sem usar drogas. A partir da avaliação das crenças relacionadas à fissura - desejo intenso de utilizar a droga - observou-se que $66,6 \% \quad(n=16)$ dos sujeitos referiram que o uso de drogas mantinha-se em função destas crenças, e 37,5\% ( $n=9)$ afirmaram que a fissura não desapareceria, a não ser com o uso de drogas.

Schafer e Brown (1991), autores do Marijuana Effects Expectancy Questionnaire (MEEQ), descrevem a origem da escala informando que os itens do questionário derivaram-se de entrevistas estruturadas e aplicadas em 108 sujeitos, 50\% homens, com idades entre 19 e 77 anos. As entrevistas foram realizadas por voluntários da comunidade de San Diego, Califórnia, com sujeitos selecionados de modo a assegurar uma grande variedade na escala de idades e um nível de exposição às drogas. Foi garantido aos participantes que suas respostas seriam confidenciais. Ao serem questionados sobre os efeitos esperados após o consumo de uma quantidade moderada de maconha, os participantes eram incentivados a responder de acordo com suas crenças ou experiências com a droga, desconsiderando se tinham realmente usado a substância; além de utilizar o seu próprio padrão para quantidade ou ajuste.

Cada entrevista foi transcrita, sendo realizada uma análise de acordo com os critérios de Nunnally (1978). As indicações similares foram agrupadas e, dos itens individuais, foram selecionados os que melhor representavam a diversidade do índice para cada área, tendo-se extremo cuidado com a redundância. Desse processo, resultaram 70 itens que refletem os efeitos da maconha.

$\mathrm{Na}$ segunda parte do estudo, Schafer e Brown (1991) submeteram o MEEQ a 704 estudantes de duas universidades da parte sul da Califórnia. Cerca de 62\% da amostra eram mulheres e a média de idade foi de 19,23 anos ( $\mathrm{SD}=3,42)$, entre 17 e 54 anos. Quanto ao estado civil, 93\% da amostra era de solteiras, 5\% vivia com um parceiro não-casado, $1 \%$ era de separadas, divorciadas ou viúvas. Quanto ao uso de substâncias, 295 estudantes (42\%) relataram usar maconha, em média 7 vezes por mês $(\mathrm{SD}=8,16)$. O MEEQ foi administrado em grupo, junto com os dados sociodemográficos e o registro habitual do uso da droga.

Aarons, Brown, Stice e Coe (2001) avaliaram as propriedades psicométricas do MEEQ num estudo longitudinal, utilizando uma amostra clínica e não-clínica de adolescentes. Confirmaram o MEEQ como uma escala confiável e fidedigna para o uso entre adolescentes (15 a 19 anos de idade). A consistência interna $(0,82)$ foi boa e semelhante aos achados de Schafer e Brown (1994), quando validaram o MEEQ com uma amostra de adultos.

Os adolescentes com maiores expectativas para prejuízo cognitivo/comportamental e efeitos globais negativos, apresentavam menor probabilidade de uso de maconha após 2 anos de estudo, sugerindo que tais expectativas podem prevenir o começo do uso de maconha no período da adolescência.

Em geral, maiores pontuações nas escalas de expectativas negativas e maiores expectativas para prejuízo cognitivo/comportamental e efeitos globais negativos estavam associadas com a cessação do uso de maconha, enquanto que menores expectativas negativas e menores expectativas de prejuízo cognitivo/comportamental e efeitos negativos associavam-se à continuidade do uso de maconha. Os achados de Aarons e colaboradores (2001) correlacionam as baixas expectativas para prejuízo cognitivo/comportamental e outros efeitos negativos, com a abertura de uma porta de entrada para o envolvimento com a substância. Porém, esses dados encontrados podem sugerir a busca de um potencial estratégico para reforços preventivos quanto ao desenvolvimento e progresso seqüencial do uso de maconha, evitando-se o uso inicial (no momento em que as expectativas negativas venham a ser trabalhadas e reforçadas, em detrimento das expectativas positivas), em relação ao uso de substâncias.

Galen e Henderson (1999) validaram o MEEQ (Schafer \& Brown, 1991), estudando uma população clínica, cuja amostra constituiu-se de 149 homens, com idades entre 23 e 59 anos, internados para tratamento numa clínica de dependência química (21 a 28 dias), o Allen Park Veterans Administration Hospital. Os não-usuários de maconha apresentaram maiores expectativas de efeitos negativos do que os usuários. Quanto às expectativas de maior relaxamento e efeitos do craving, os usuários apresentaram maiores pontuações do que os não-usuários ou os que pararam de usar. Os achados sugerem também uma modificação da teoria que apresenta as expectativas negativas como fatores capazes de evitar o início do uso em não-usuários de drogas, pois esse estudo demonstrou que os usuários de maconha apresentam expectativas negativas globais mais baixas quando comparados aos não-usuários.

Psico-USF, v. 9, n. 2, p. 129-136, Jul./Dez. 2004 
Nesta pesquisa, para estudar-se as expectativas de resultados relacionadas ao uso de maconha, realizouse extensa busca na literatura, onde foram encontradas escalas de expectativas de resultados relacionadas ao uso de álcool e cocaína, já validadas, sendo que nenhum instrumento específico para maconha, já validado no Brasil, foi encontrado. Durante essas buscas, descobriuse o Marijuana Effects Expectancy Questionnaire (MEEQ; Shaffer \& Brown, 1991), uma escala americana validada pelos próprios autores. O instrumento foi solicitado e obteve-se a nova versão reestruturada, o Marijuana Expectancy Questionnaire (MEQ; Schafer \& Brown, 1994), enviada pelos autores, com a devida autorização para tradução, adaptação cultural e equivalência semântica para uma população brasileira.

A divulgação de pesquisas envolvendo a validação e as propriedades psicométricas de escalas traduzidas e adaptadas ao nosso idioma e país ainda é restrita, embora o seu uso seja disseminado. Porém, não basta traduzir um instrumento, é necessário realizar uma avaliação rigorosa dessa tradução, verificando a adaptação transcultural e suas propriedades psicométricas (Ciconelli, 2003; Pasquali, 1998).

As crenças, atitudes, costumes, comportamentos e hábitos sociais são característicos de cada sociedade e ditam os comportamentos que se refletem na cultura de um povo; por isso, a tradução de uma escala deve primar pela linguagem simples e clara, mantendo-se equivalente no que tange aos seus conceitos culturais (Ciconelli, Ferraz, Santos, Meinão \& Quaresma, 1999). Metodologicamente, a tradução de um instrumento deve ser feita por, pelo menos, dois tradutores, independentes e cientes dos objetivos da tradução. A tradução inicial deve ser avaliada, realizando-se após isso o processo back-translation; ou seja, o instrumento deve ser vertido outra vez para o idioma original, e o resultado disso deve ser comparado ao instrumento de origem, sendo que esta etapa deve ser feita por outros dois tradutores com fluência nos dois idiomas, pois a tradução deve ser feita para a língua materna dos tradutores (que ao contrário dos primeiros, não devem conhecer o objetivo da tradução). Logo após, um comitê multidisciplinar, com especialistas, conhecedores da finalidade do instrumento e dos conceitos a serem analisados, deve produzir a versão final do instrumento, baseado nas várias traduções e back-translation, comparando os resultados entre si (Duarte, Miyazaki, Ciconelli \& Sesso, 2003; Guillemin, Bombardier \& Beaton, 1993).

Menezes (1998), citando Morley e Snaith (1989), apresenta três aspectos de validade que podem ser avaliados operacionalmente: 1) Validade de conteúdo: este termo refere-se ao julgamento do instrumento quanto à abrangência dos diferentes aspectos do seu objeto, verificando se não há elementos que possam ser atribuídos a outros objetos; 2) Validade de critério: avalia o grau com que o instrumento discrimina entre pessoas que diferem em determinada(s) característica(s) de acordo com um critério padrão; 3) Validade de construto: refere-se à demonstração de que o instrumento realmente mede aqui o que ele se propõe a medir.

A equivalência semântica trata de avaliar a equivalência gramatical e de vocabulário, pois muitas palavras de um determinado idioma não possuem tradução adequada para outro. A equivalência idiomática trata das dificuldades em traduzir expressões coloquiais de um determinado idioma. A equivalência experimental ou cultural prioriza que os termos utilizados sejam coerentes com as experiências vivenciadas pela população à qual se destina. A equivalência conceitual trata de adequar os conceitos dos termos utilizados; por exemplo, num contexto cultural "irmão" ou "tia" podem significar mais do que um grau de parentesco (Ciconelli, 1997).

A análise teórica dos itens consiste em pedir opiniões de outras pessoas, as quais ainda não constituem a amostra representativa da população na qual $\mathrm{O}$ instrumento será validado. Trata-se de uma análise teórica dos itens mediante a análise semântica e análise feita por juízes, antes de partir para o estudo piloto. A análise semântica dos itens verifica se todos os itens são compreensíveis para todos os membros da população à qual o instrumento se destina. Pode-se aplicar o instrumento numa amostra de 30 pessoas da população-meta e, em seguida, discutir as dúvidas que forem suscitadas. Ainda podem-se checar os itens em pequenos grupos de pessoas (3 ou 4) numa situação de brainstorming, na qual se apresenta item por item, para que cada um do grupo o reproduza. Se a reprodução do item é feita, o item é corretamente compreendido. Senão, as próprias pessoas, junto ao pesquisador, irão sugerir como deveria ser formulado o item. Já a análise dos juízes consiste em peritos na área do construto ajuizarem se os itens estão se referindo ou não ao traço em questão (Pasquali, 1998).

$\mathrm{Na}$ adaptação e validação de uma escala mede-se a confiabilidade utilizando-se o coeficiente alfa de Cronbach, que verifica a homogeneidade dos componentes do instrumento, ou seja, a consistência interna dos itens. Avalia-se o critério de equivalência cultural da escala diversificandose a amostra e obtendo-se um número abrangente de participantes (Cazorla, Silva \& Vendramini, 1999).

Apresentar simplesmente a versão traduzida de um instrumento nada significa. É necessário comprovar sua adequação e adaptação ao contexto cultural da amostra em questão. Isso significa conhecer sua estrutura fatorial, consistência interna e poder de discriminação entre amostras clínicas e não-clínicas (Gouveia, Duarte, \& Seminotti, 1999). Neste estudo, realizou-se um processo de tradução, adaptação cultural e equivalência semântica da escala Marijuana Expectancy Questionnaire (MEQ; Schafer \& Brown, 1994), para uma população brasileira. 


\section{Método}

\section{Participantes}

No processo de equivalência semântica do MEQ (Schafer \& Brown, 1994), descrito neste estudo, 10 sujeitos, do sexo masculino e feminino, entre 12 e 55 anos de idade e com a escolaridade mínima da 5a série do ensino fundamental, responderam a primeira tradução realizada do instrumento. Essa amostra de 10 participantes caracterizou-se por universitários, sujeitos da população geral, usuários de maconha ou não, usuários de maconha internados para tratamento de dependência química e adolescentes usuários de maconha encaminhados pelo Ministério Público de Porto Alegre-RS.

Um grupo de 4 sujeitos, sendo 2 psicólogos, com conhecimento em construção e validação de escalas psicológicas e dois estudantes (ensino fundamental e ensino médio) compuseram o brainstorming, onde o instrumento foi analisado item a item. Foram excluídos do estudo os sujeitos sem a escolaridade mínima estabelecida ou sem condições de entender o que estava sendo solicitado no preenchimento dos instrumentos de pesquisa.

\section{Instrumentos}

Entrevista estruturada, contendo os dados sociodemográficos e critérios diagnósticos para dependência química, de acordo com o DSM-IV-R.

Marijuana Expectancy Questionnaire (MEQ; Schafer \& Brown, 1994). Uma escala Likert estruturada com 78 itens, relacionados ao uso de maconha, onde são atribuídos pontos de 1 (discordo totalmente) a 5 (concordo totalmente), distribuídos em 6 subescalas: prejuízo cognitivo e comportamental $(\mathrm{N}=13)$, redução de tensão e relaxamento $(\mathrm{N}=9)$, facilitação social e sexual $(\mathrm{N}=10)$, aumento de percepção e cognição $(\mathrm{N}=9)$, efeitos globais negativos $(\mathrm{N}=10)$, craving e efeitos físicos $(\mathrm{N}=6)$. Apesar da escala possuir 78 itens, somente 57 itens são computados para a pontuação das subescalas, em razão dos resultados encontrados na validação original, realizada pelos autores (Schafer \& Brown, 1994).

\section{Procedimento para validação semântica}

O projeto de pesquisa foi aprovado pelo Comitê de Ética em Pesquisa da PUCRS e o instrumento, o Marijuana Expectancy Questionnaire (MEQ; Schafer \& Brown, 1994) foi traduzido da língua inglesa para a língua portuguesa. $O$ trabalho foi realizado por uma profissional graduada em Letras, habilitada em língua inglesa e conhecedora do objetivo da tradução. $O$ MEQ foi aplicado em 10 sujeitos universitários, com o perfil da amostra pesquisada, a fim de verificar dúvidas, expressões confusas ou ambíguas. Todos compreenderam o que estava sendo proposto e responderam ao questionário entre 10 e 20 minutos.
Brainstorming. Reuniram-se 4 sujeitos (2 psicólogos e 2 estudantes) e apresentou-se o instrumento, item a item, para que cada um do grupo o reproduzisse verbalmente. Todos os 78 itens foram reproduzidos sem dificuldade, o que pode significar que foram corretamente compreendidos.

Back-translation. A partir da primeira tradução e do brainstorming, o instrumento foi vertido para o idioma de origem. O trabalho foi realizado por um nativo de língua inglesa, com fluência em língua portuguesa e desconhecedor do objetivo da tradução.

Tradução do instrumento. Com base no processo back-translation, o MEQ foi vertido para a língua portuguesa. O trabalho foi realizado por uma psicóloga, com fluência na língua inglesa, conhecedora do objetivo da tradução.

Comitê de juires especialistas. Todo o processo de tradução e back-translation foi encaminhado para um comitê, formado por uma equipe multidisciplinar especializada, conhecedora do tema pesquisado, da finalidade do instrumento e dos conceitos a serem analisados. $\mathrm{O}$ trabalho dos 10 juízes consistiu em detectar possíveis divergências nas traduções, cabendo-lhes comparar os resultados entre si, verificando se os itens da escala referiam-se ou não ao tema que mensuravam. Tomando por base os pareceres, produziu-se a versão final do instrumento.

\section{Resultados}

Os resultados obtidos na análise interjuízes encontram-se expressos na Tabela 1. Nesta tabela, explicita-se o parecer de cada juiz quanto às traduções do MEQ, indicando qual a versão julgou ser a mais adequada, devendo assim ser mantida na versão final do instrumento, a adaptação brasileira. Para a equivalência semântica do MEQ foi utilizada uma análise descritiva, verificando-se a freqüência e a porcentagem, apresentadas pela análise dos juízes, quanto à primeira tradução e o back-translation. O banco de dados foi estruturado e analisado no software estatístico SPSS versão 11.5. A análise estatística descritiva, encontrou uma concordância entre os dez juízes de, no mínimo, 80\% entre 57,7\% dos itens examinados, quanto à primeira tradução do MEQ. Utilizou-se o critério de decisão sobre a pertinência do item conforme Pasquali (2001).

Os resultados das avaliações de tradução e backtranslation, com os pareceres dos juízes especialistas, indicaram que não houve dificuldade de tradução nos itens do MEQ; porém, alguns ajustes foram feitos, no sentido de melhor adequar as frases traduzidas para a língua portuguesa coloquial. Todas as modificações são apresentadas a seguir. Dos 78 itens, 29 não sofreram qualquer modificação a partir da primeira tradução ou da tradução back-translation.

Psico-USF, v. 9, n. 2, p. 129-136, Jul./Dez. 2004 
Tabela 1 - Análise interjuízes: freqüência e porcentagem

\begin{tabular}{|c|c|c|c|c|c|c|c|c|c|}
\hline Itens & $\begin{array}{l}1^{\mathrm{a}} \\
\text { Tradução }\end{array}$ & $\%$ & $\begin{array}{l}\text { Back- } \\
\text { translation }\end{array}$ & $\%$ & Itens & $\begin{array}{l}1^{\mathrm{a}} \\
\text { Tradução }\end{array}$ & $\%$ & $\begin{array}{l}\text { Back- } \\
\text { translation }\end{array}$ & $\%$ \\
\hline 1 & 6 & $60 \%$ & 4 & $40 \%$ & 40 & 7 & $70 \%$ & 3 & $30 \%$ \\
\hline 2 & 8 & $80 \%$ & 2 & $20 \%$ & 41 & 9 & $90 \%$ & 1 & $10 \%$ \\
\hline 3 & 6 & $60 \%$ & 4 & $40 \%$ & 42 & 9 & $90 \%$ & 1 & $10 \%$ \\
\hline 4 & 10 & $100 \%$ & 0 & $0 \%$ & 43 & 7 & $70 \%$ & 3 & $30 \%$ \\
\hline 5 & 9 & $90 \%$ & 1 & $10 \%$ & 44 & 10 & $100 \%$ & 0 & $0 \%$ \\
\hline 6 & 9 & $90 \%$ & 1 & $10 \%$ & 45 & 9 & $90 \%$ & 1 & $10 \%$ \\
\hline 7 & 7 & $70 \%$ & 3 & $30 \%$ & 46 & 8 & $80 \%$ & 2 & $20 \%$ \\
\hline 8 & 9 & $90 \%$ & 1 & $10 \%$ & 47 & 9 & $90 \%$ & 1 & $10 \%$ \\
\hline 9 & 7 & $70 \%$ & 3 & $30 \%$ & 48 & 7 & $70 \%$ & 3 & $30 \%$ \\
\hline 10 & 9 & $90 \%$ & 1 & $10 \%$ & 49 & 9 & $90 \%$ & 1 & $10 \%$ \\
\hline 11 & 6 & $60 \%$ & 4 & $40 \%$ & 50 & 9 & $90 \%$ & 1 & $10 \%$ \\
\hline 12 & 6 & $60 \%$ & 4 & $40 \%$ & 51 & 7 & $70 \%$ & 3 & $30 \%$ \\
\hline 13 & 9 & $90 \%$ & 1 & $10 \%$ & 52 & 8 & $80 \%$ & 2 & $20 \%$ \\
\hline 14 & 5 & $50 \%$ & 5 & $50 \%$ & 53 & 10 & $100 \%$ & 0 & $0 \%$ \\
\hline 15 & 7 & $70 \%$ & 3 & $30 \%$ & 54 & 6 & $60 \%$ & 4 & $40 \%$ \\
\hline 16 & 8 & $80 \%$ & 2 & $20 \%$ & 55 & 8 & $80 \%$ & 2 & $20 \%$ \\
\hline 17 & 8 & $80 \%$ & 2 & $20 \%$ & 56 & 8 & $80 \%$ & 2 & $20 \%$ \\
\hline 18 & 7 & $70 \%$ & 3 & $30 \%$ & 57 & 8 & $80 \%$ & 2 & $20 \%$ \\
\hline 19 & 8 & $80 \%$ & 2 & $20 \%$ & 58 & 9 & $90 \%$ & 1 & $10 \%$ \\
\hline 20 & 8 & $80 \%$ & 2 & $20 \%$ & 59 & 7 & $70 \%$ & 3 & $30 \%$ \\
\hline 21 & 6 & $60 \%$ & 4 & $40 \%$ & 60 & 9 & $90 \%$ & 1 & $10 \%$ \\
\hline 22 & 6 & $60 \%$ & 4 & $40 \%$ & 61 & 8 & $80 \%$ & 2 & $20 \%$ \\
\hline 23 & 6 & $60 \%$ & 4 & $40 \%$ & 62 & 9 & $90 \%$ & 1 & $10 \%$ \\
\hline 24 & 10 & $100 \%$ & 0 & $0 \%$ & 63 & 7 & $70 \%$ & 3 & $30 \%$ \\
\hline 25 & 8 & $80 \%$ & 2 & $20 \%$ & 64 & 6 & $60 \%$ & 4 & $40 \%$ \\
\hline 26 & 10 & $100 \%$ & 0 & $0 \%$ & 65 & 7 & $70 \%$ & 3 & $30 \%$ \\
\hline 27 & 7 & $70 \%$ & 3 & $30 \%$ & 66 & 6 & $60 \%$ & 4 & $40 \%$ \\
\hline 28 & 9 & $90 \%$ & 1 & $10 \%$ & 67 & 9 & $90 \%$ & 1 & $10 \%$ \\
\hline 29 & 7 & $70 \%$ & 3 & $30 \%$ & 68 & 9 & $90 \%$ & 1 & $10 \%$ \\
\hline 30 & 6 & $60 \%$ & 4 & $40 \%$ & 69 & 8 & $80 \%$ & 2 & $20 \%$ \\
\hline 31 & 7 & $70 \%$ & 3 & $30 \%$ & 70 & 7 & $70 \%$ & 3 & $30 \%$ \\
\hline 32 & 7 & $70 \%$ & 3 & $30 \%$ & 71 & 7 & $70 \%$ & 3 & $30 \%$ \\
\hline 33 & 8 & $80 \%$ & 2 & $20 \%$ & 72 & 10 & $100 \%$ & 0 & $0 \%$ \\
\hline 34 & 8 & $80 \%$ & 2 & $20 \%$ & 73 & 8 & $80 \%$ & 2 & $20 \%$ \\
\hline 35 & 10 & $100 \%$ & 0 & $0 \%$ & 74 & 8 & $80 \%$ & 2 & $20 \%$ \\
\hline 36 & 10 & $100 \%$ & 0 & $0 \%$ & 75 & 7 & $70 \%$ & 3 & $30 \%$ \\
\hline 37 & 7 & $70 \%$ & 3 & $30 \%$ & 76 & 10 & $100 \%$ & 0 & $0 \%$ \\
\hline 38 & 10 & $100 \%$ & 0 & $0 \%$ & 77 & 8 & $80 \%$ & 2 & $20 \%$ \\
\hline 39 & 7 & $70 \%$ & 3 & $30 \%$ & 78 & 6 & $60 \%$ & 4 & $40 \%$ \\
\hline
\end{tabular}

Nos itens $1,6,8,9,10,14,16,21,25,43,47$, $50,51,55,56,57,58,59,61,63,67,70,71,72,76$ e 78 houve a inclusão do gênero feminino entre parênteses, para facilitar a identificação do participante com a frase apresentada. No item 2, o verbo tornar foi substituído por parecer, visando melhor adequação na tradução. No item 5, a tradução foi adaptada e craving foi traduzido como fissura, sendo colocada uma explicação entre parênteses para facilitar o entendimento do vocábulo.

No item 14, a expressão costumo não me enturmar foi substituída por isolado. Nos itens 15, 16, 17, 20, 23, $26,31,32,35,41,45,46,50,54,61,65,69,70,73$ e 76 a frase foi invertida para a ordem direta, de acordo com a gramática clássica da língua portuguesa, visando melhor entendimento da frase. No item 21, o termo técnico paranóico foi substituído pelo termo coloquial desconfiado, sendo que o primeiro permaneceu na frase, entre parênteses.

No item 28 , o verbo retrair foi substituído por isolar. Nos itens 30, 34, 52, 64 e 74 foram incluídas explicações, entre parênteses. No item 31, distante foi substituído 
por perdido, para melhor adequação na tradução. Nos itens 37, 38 e 40 foi realizada a correção nas traduções.

Alguns vocábulos foram substituídos por sinônimos, para melhor adequação na realidade da populaçãoalvo da pesquisa, conforme a sugestão do comitê de especialistas. Foi o caso o item 37, onde o vocábulo zumbido foi substituído por zunido; item 47, onde desastrado foi substituído por descuidado; item 59, onde deprimido foi substituído por pra baixo; item 69, onde nervoso foi substituído por inquieto e itens 70 e 78 , onde a expressão relaxado em grupos foi substituída por situações sociais.

Nos itens 5, 21, 30, 34, 43, 52, 64 e 74 foram incluídas explicações, entre parênteses, para facilitar o entendimento da frase ou de alguns termos técnicos. Houve um consenso entre os especialistas na manutenção das palavras de uso comum na língua portuguesa.

Foi sugerido pelos especialistas que no item 5, craving fosse traduzido como "fissura", embora a primeira tradução tenha sido feita como "desejo intenso", ocasionando a mesma tradução no back-translation. A equivalência idiomática foi realizada no item 64, no qual munchies foi traduzido como larica, uma gíria comum entre usuários ou dependentes químicos. A equivalência conceitual dos itens, ou seja, a validade do conceito explorado e os eventos experimentados pela pessoa foram obtidas em todos o itens, pois os conceitos da versão original não se diferenciavam dos conceitos da cultura-alvo.

\section{Discussão dos resultados}

As expectativas de resultados relacionadas ao uso de maconha poderão ser a chave para intervenções que minimizem esse uso ou que até mesmo o previnam (Brown, 1993; Marlatt \& Gordon, 1993). No entanto, verifica-se que esse construto não tem sido muito estudado e pesquisado pela comunidade científica, como denotam os indexadores onde foram realizadas buscas de trabalhos empíricos. No Brasil, nos sites de buscas e nas bibliotecas procuradas também não foi encontrada uma escala para avaliar crenças específicas para maconha.

O MEEQ (1991) foi a única escala encontrada que avaliava as crenças específicas relacionadas à maconha, daí a motivação para traduzi-la e validá-la para uma amostra brasileira. Logo após o contato com os autores, recebeu-se a autorização e a cópia da escala em sua versão mais recente, o MEQ (1994). Priorizouse a qualidade do processo de tradução, reduzindo a probabilidade de erros e interpretações que divergissem do texto original (Ciconelli, 2003; Pasquali, 1998).

Por meio desse estudo, verificou-se que as etapas de tradução da versão original do MEQ para o português, não apresentaram dificuldades. A equivalência conceitual foi obtida facilmente nas duas versões. As comparações entre traduções e back-translation resultaram apenas em adaptações culturais referentes às expressões idiomáticas e coloquiais (Cazorla, Silva \& Vendramini, 1999; Ciconelli, 1997; Duarte, Miyazaki, Ciconelli \& Sesso, 2003).

$O$ processo de validação semântica foi criterioso, o que pode ter facilitado a tradução e adequação do instrumento à língua portuguesa (Duarte, Miyazaki, Ciconelli \& Sesso, 2003; Guillemin, Bombardier \& Beaton, 1993). A equivalência conceitual pode ser obtida pelo consenso entre os participantes do brainstorming e, posteriormente, pela análise dos juízes (Ciconelli, 2003; Pasquali, 1998). Com base nesses resultados, construiu-se, então, a versão final do Marijuana Expectancy Questionnaire (MEQ) - adaptação brasileira.

\section{Conclusões}

Sem uma metodologia adequada para o processo de tradução, adaptação cultural e equivalência semântica de um instrumento de pesquisa, podem ocorrer problemas, no sentido da tradução ser tendenciosa ou diferente da versão original. Quanto à adaptação cultural, os hábitos e as atividades de uma população devem ser levados em conta, para que o instrumento torne-se válido.

Se o método de validação não for adequado, os resultados tendem a ser equivocados, podendo prejudicar as pessoas caso o tratamento se baseie em achados de pesquisa. Portanto, é importante que os pesquisadores relatem todas as etapas da tradução e detalhem os resultados encontrados, a fim de que as escolhas pelo instrumento possibilitem avaliar os objetivos de seu estudo.

A versão final do MEQ (Schafer \& Brown, 1994) conservou o nome original em inglês e acrescentou-se "adaptação brasileira". Foi aplicada num estudo piloto, numa amostra de 30 participantes, e mostrou-se de fácil aplicação. No momento, a validação psicométrica está sendo realizada, sendo que nesta etapa a amostra deverá atingir o número de 400 participantes.

O instrumento, após a validação geral, será importante para clínicos e pesquisadores, que ainda não dispõem de uma escala específica para avaliar crenças acerca da maconha. Mediante a verificação das crenças, evidenciadas nos escores pontuados nas subescalas do instrumento, os profissionais poderão identificar, eventualmente, a intervenção necessária para o tratamento e prevenção do uso e abuso de maconha.

O presente estudo tem um papel importante na área da dependência química, especificamente no uso e abuso de maconha, somando-se aos instrumentos que avaliam outras drogas, como o álcool e a cocaína.

\section{Referências}

Aarons, G. A., Brown, S. A., Stice, E. \& Coe, M. T. (2001). Psychometric evaluation of the marijuana and Psico-USF, v. 9, n. 2, p. 129-136, Jul./Dez. 2004 
stimulant effect expectancy questionnaires for adolescents. Addictive Behaviours, 26, 219-236.

Beck, A. T., Wrigh, F. D., Newman, C. F. \& Liese, B. S. (1993). Cognitive therapy of substance abuse. EUA: The Guilford Press.

Brown, S. A. (1993). Drug effect expectancies and addictive behavior change. Experimental and Clinical Psychopharmacology, 1, 55-67.

Cazorla, I. M., Silva, C. B. \& Vendramini, C. (1999). Adaptação e Validação de uma escala de atitudes em relação à estatística. Atas da Conferência Internacional "Experiências e expectativas do ensino de estatistica-desafios para o século XXT'. Florianópolis, SC.

Cicconelli, R. M. (1997). Tradução para o português e validação do questionário genérico de avaliação de qualidade de vida Medical Outcomes Study 36 - item short-form bealth survey (SF-36) (Tese de Doutorado). São Paulo: Universidade Federal de São Paulo - Escola Paulista de Medicina.

Ciconelli, R. M. (2003). Medidas de avaliação de qualidade de vida. Revista Brasileira de Reumatologia, 43, 9-13.

Ciconelli, R. M., Ferraz, M. B., Santos, W., Meinão, I. \& Quaresma, M. R. (1999). Tradução para a língua portuguesa e validação do questionário genérico de avaliação de qualidade de vida SF-36 (Brasil SF-36). Revista Brasileira de Reumatologia, 39,143-150.

Duarte, P. S., Miyazaki, M. C. O. S., Ciconelli, R. M. \& Sesso, R. (2003). Tradução e adaptação cultural do instrumento de avaliação de qualidade de vida para pacientes renais crônicos (KDQOL-SF TM). Revista da Associação Médica Brasileira, 49, 375-381.

Eiser, J. R. (1997). Attitudes and beliefs. Em A. Baum, C. McManus, S. Newman, J. Weinman \& R. West (Orgs.). Cambridge handbook of psychology, health and medicine (pp. 3-7). Cambridge: Cambridge University Press.

Galen, Luke W. \& Henderson, Melinda J. (1999). Validation of cocaine and marijuana effect expectancies in a treatment setting. Addictive Behaviors, 24, 719-724.

Gouveia, V. V., Duarte, L. R. S. \& Seminotti, R. P.
(1999). Inventário de mobilidade para avaliar agorafobia, IM: adaptação brasileira. Psico, 30, 141-159.

Guillemin, F., Bombardier C. \& Beaton, D. (1993). Cross-cultural adaptation of health-related quality of life measures: literature review and proposed guidelines. Journal Clinical Epidemiology, 46, 14-32.

Laranjeira, R., Jungerman, F. \& Dunn, J. (1998). Drogas: maconha, cocaína e crack. Coleção Mitos e Verdades. São Paulo: Contexto.

Marlatt, A. \& Gordon, J. (1993). Prevenção da recaída: estratégia e manutenção no tratamento de comportamentos aditivos. Porto Alegre: Artes Médicas.

Menezes, P. R. (1998).Validade e confiabilidade das escala de avaliação em psiquiatria. Revista de Psiquiatria Clínica, 25, 214-216.

Morley, S. \& Snaith, P. (1989). Principles of psychological assessment. Em Freman, C. \& Tyrer, P. (Orgs.). Research methods in psychiatry. London: Gaskell.

Nunnally, J. C. (1978). Psychometric theory. New York: McGraw-Hill.

Oliveira, M. S., Szupzsynski, K. P. D. R., Camilo, R. \& Faggiani, F. (2003). O estudo das crenças de adolescentes usuários de maconha. Anais do IV Congresso da Sociedade Brasileira de Terapias Cognitivas. João Pessoa, PB, 153.

Pasquali, L. (1998). Princípios de elaboração de escalas psicológicas. Revista de Psiquiatria Clínica, 25, 206-213.

Pasquali, L. (2001). Técnicas de Exame Psicológico - TEP manual: fundamentos das técnicas psicológicas. São Paulo: Casa do Psicólogo.

Schafer, J. \& Brown, S. A. (1991). Marijuana and cocaine effect expectancies and drug use patterns. Journal of Conculting and Clinical Psychology, 59, 558-565.

Schafer, J. \& Brown, S. A. (1994). Marijuana Expectancy Questionnaire (MEQ).

Recebido em junbo de 2004 Reformulado em outubro de 2004 Aprovado em novembro de 2004

Sobre os autores:

Rosemeri Siqueira Pedroso é psicóloga e mestranda em Psicologia Clínica pela PUCRS.

Margareth da Silva Oliveira é psicóloga, doutora em Ciências da Saúde pela UNIFESP e professora no Programa de Pós-Graduação em Psicologia da PUCRS. 
Renata Brasil Araujo é psicóloga, doutoranda em Psicologia pela PUCRS, coordenadora do Programa de Dependência Química do Hospital Psiquiátrico São Pedro (HPSP) e professora da Clínica Wainer \& Piccoloto Centro de Terapia Cognitivo-Comportamental.

João Feliz Duarte de Moraes é matemático e estatístico, doutor em Gerontologia Biomédica pela PUCRS, e professor do Departamento de Estatística da PUCRS e da UFRGS, na graduação e nos Programas de PósGraduação de Psicologia, Odontologia e Medicina. 